\title{
Noniridescent Biomimetic Photonic Microdomes by Inkjet Printing
}

\author{
Ravi Shanker, Samim Sardar, Shangzhi Chen, Sampath Gamage, Stefano Rossi, and Magnus P. Jonsson*
}

Cite This: Nano Lett. 2020, 20, 7243-7250

Read Online

ABSTRACT: Certain bird species have evolved spectacular colors that arise from organized nanostructures of melanin. Its high refractive index $(\sim 1.8)$ and broadband absorptive properties enable vivid structural colors that are nonsusceptible to photobleaching. Mimicking natural melanin structural coloration could enable several important applications, in particular, for noniridescent systems with colors that are independent of incidence angle. Here, we address this by forming melanin photonic crystal microdomes by inkjet printing. Owing to their curved nature, the microdomes exhibit noniridescent vivid structural coloration, tunable throughout the visible range via the size of the nanoparticles. Large-area arrays $\left(>1 \mathrm{~cm}^{2}\right)$ of high-quality photonic microdomes could be printed on both rigid and flexible substrates.

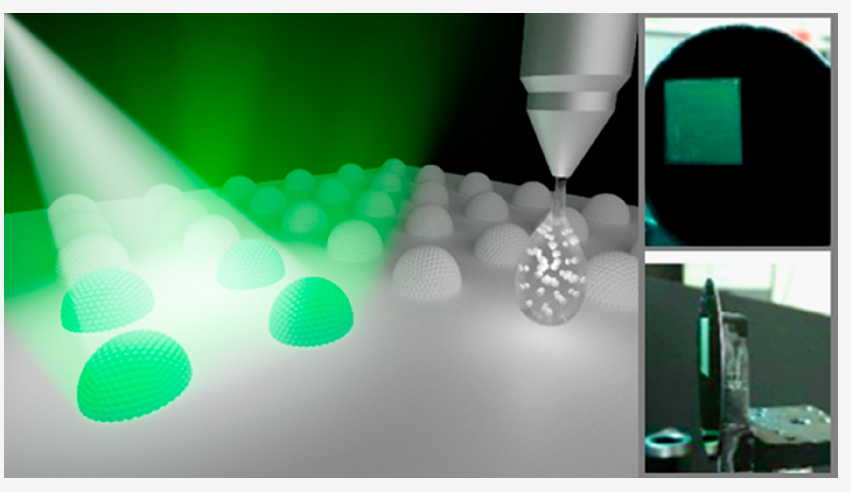
Combined with scalable fabrication and the nontoxicity of melanin, the presented photonic microdomes with noniridescent structural coloration may find use in a variety of applications, including sensing, displays, and anticounterfeit holograms.

KEYWORDS: Structural colors, Photonic crystals, Melanin, Inkjet printing, RGB pixels, Confined self-assembly

$\mathrm{N}$ ature provides a myriad examples of living organisms that have evolved intricate ordered nanostructures that produce beautiful colors via light interference instead of material absorption. ${ }^{1,2}$ Such natural structural coloration (SC) has inspired the development of engineered nanophotonic materials, which circumvent limitations of traditional coloration strategies based on pigments or dyes. ${ }^{3,4}$ Not least, organic dyes tend to fade over time, and inorganic pigments are often based on toxic heavy metals, with harmful effects on the environment and human beings. ${ }^{5}$

Although there are many top-down fabrication methods to prepare artificial SC devices, bottom-up approaches are preferred for most practical applications due to scalability and low cost. $^{6-11}$ In particular, SC systems have been commonly prepared by convective self-assembly of spherical polymeric or silica nanoparticles (NPs) into three-dimensional planar thin film photonic crystals. ${ }^{12,13}$ These colloidal crystals produce iridescent colors, which means that they reflect different colors at different angles. This limits their suitability for many applications, such as color displays with wide viewing angles or photonic paints. Previous approaches to reduce the angular dependence of SC include fabrication of nonplanar systems and short-range-ordered amorphous assemblies of NPs. ${ }^{14,15}$ However, color saturation is often limited due to incoherent light scattering that contributes to a broad background signal. ${ }^{16}$ To suppress such white background and improve color hue, researchers have incorporated lightabsorbing materials such as carbon black into colloidal NP assemblies. ${ }^{17-19}$ An alternative approach, which avoids the need for black additives and thereby facilitates better control of the self-assembly process, uses NPs made of partially lightabsorbing materials, such as melanin. In this way, the NPs themselves contribute to suppressing incoherent scattering. ${ }^{20-22}$ Interestingly, similar systems can be found in Nature via Avian birds that produce SC using melanin NPs (MNPs). ${ }^{23,24}$

From an application point of view, it is desirable to enable well-controlled and precise patterns of noniridescent SC, for which inkjet printing of NP-containing droplets that selfassemble into microdomes is a promising approach. ${ }^{15}$ Inkjet printing provides precise and reproducible deposition of small microdroplets with diameters down to only a few micrometers. ${ }^{25}$ The technique also offers flexibility in creating high spatial resolution patterns over large areas at low cost and low material usage, which open avenues for wide-ranging applications in sensing, encryption, and displays. ${ }^{26,27}$ On the other hand, printing of NP inks that form suitable microdroplets that also self-assemble into microdomes with high NP order is challenging as it requires several different aspects to be jointly fine-tuned. To our knowledge, this was not yet achieved for melanin-based systems.

Received: June 23, 2020

Revised: September 15, 2020

Published: September 16, 2020 
In this work, we demonstrate inkjet printing of photonic MNP microdomes with high order (schematically depicted in Figure 1a). The microdomes provide highly vivid SC that is

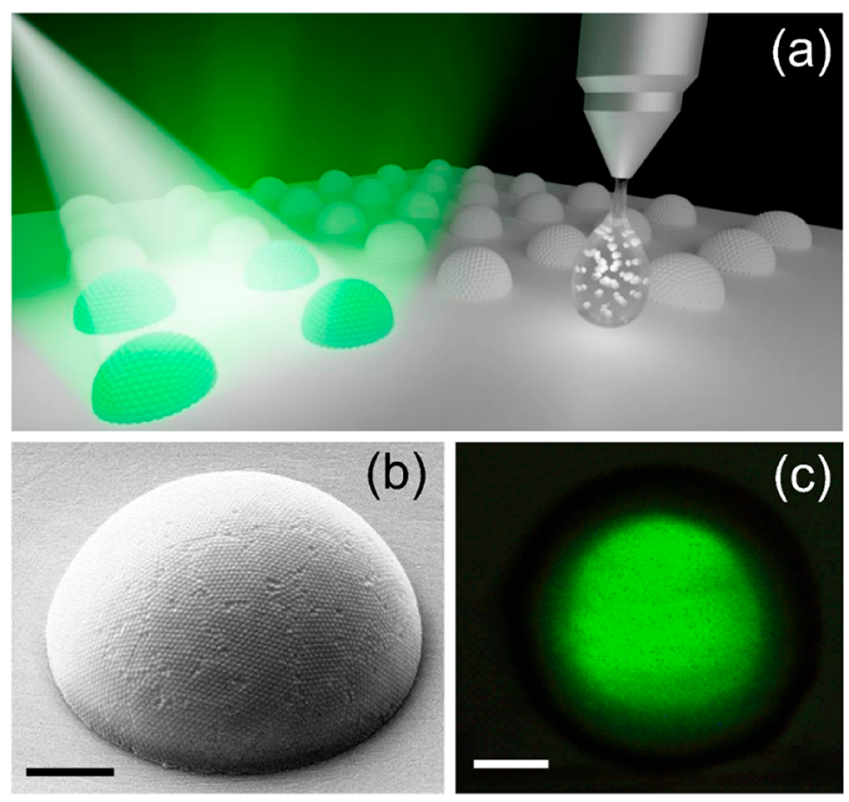

Figure 1. (a) Schematic illustration of inkjet-printed MNP photonic microdomes. (b) Scanning electron microscopy image of a typical inkjet-printed MNP photonic microdome (tilted at $54^{\circ}$ ). (c) Optical microscopy bright-field image showing green SC from a typical inkjetprinted MNP microdome. The scale bars in (b) and (c) are $3 \mu \mathrm{m}$.

noniridescent. We synthesized MNPs of different sizes (around 100-300 nm) and formulated printable inks using a high boiling point binary cosolvent, allowing stable jetting with high printing efficiency and spatial uniformity. Solvent evaporation resulted in confined self-assembly of MNPs into microdomes with high order and bright SC, as exemplified in Figure 1b,c, respectively. Varying MNP size enabled printing of red, green, and blue (RGB) photonic microdome pixels, as studied in detail by microreflectance spectroscopy, optical microscopy, and electron microscopy. We demonstrate inkjet printing of large-area arrays $\left(>1 \mathrm{~cm}^{2}\right)$ of photonic MNP microdomes and show that they provide bright and highly noniridescent SC, not only for specular reflection but also for diffusive and backscattered light. Printing was successful on various substrates, including on flexible polydimethylsiloxane (PDMS) and rigid silicon wafers.

We prepared MNPs by polymerizing dopamine hydrochloride monomers in a water-methanol solution, as illustrated in Figure $S 1$ and explained in detail in the experimental section of the Supporting Information (SI). We could accurately tune the size of the MNPs by changing the monomer concentration, enabling MNPs from approximately 110 to $260 \mathrm{~nm}$ (red squares in Figure 2a). The scanning electron microscopy (SEM) image in the inset of Figure $2 \mathrm{a}$ confirms that the MNPs are spherical and uniform in size. Indeed, the size distribution in terms of coefficient of variation (CV) is below $10 \%$ for all particle sizes (blue circles in Figure 2a). Such low polydispersity is key to create SC by selfassembly into photonic crystals. ${ }^{28}$ The MNPs showed a clear negative zeta-potential (average $-38 \pm 2 \mathrm{mV}$ ), which results in electrostatic repulsion between MNPs and thereby led to stable MNP dispersions.
To be compatible with inkjet printing, the inks need suitable rheological properties, which are related to the Reynolds $\left(R_{\mathrm{e}}\right)$ and Ohnesorge $\left(O_{\mathrm{h}}\right)$ numbers: ${ }^{29}$

$$
\begin{aligned}
& R_{\mathrm{e}}=\frac{\rho v d}{\eta} \\
& O_{\mathrm{h}}=\frac{\eta}{\sqrt{\gamma \rho d}}
\end{aligned}
$$

where $\rho, \eta$, and $\gamma$ are density, viscosity, and surface tension of the inks, respectively. $v$ is the droplet velocity, and $d$ is the nozzle diameter. Both $R_{\mathrm{e}}$ and $O_{\mathrm{h}}$, and thereby printing performance, can be tuned by altering the surface tension and viscosity of the solvent. ${ }^{30}$ After synthesis, the MNPs are dispersed in water, which is not suitable for inkjet printing due to low viscosity and high surface tension. We therefore modified the solutions using a binary solvent of high viscosity (FAEG, a binary mixture of formamide and ethylene glycol) and added a surfactant (Brij 30) to decrease surface tension. The presence of surfactant may also contribute to Marangoni flows to facilitate NP ordering and suppress crack formation. ${ }^{31-33}$ The binary FAEG solvent also has a low evaporation rate, which can aid the self-assembly process by providing the MNPs with sufficient time to form close-packed order in each microdroplet before complete evaporation. Figure $2 \mathrm{~b}$ shows that both viscosity and surface tension of the MNP inks could be varied by changing the solvent to MNP solution ratio (solvent/MNP sol ratio) and surfactant concentration, respectively. For solvent/MNP sol ratios up to 1 , we used only formamide as solvent, whereas higher ratios correspond to using the binary solvent (see the experimental section in the SI for details).

In order to determine suitable concentrations for ink formulation, we construct a phase diagram with $R_{\mathrm{e}}$ and $O_{\mathrm{h}}$ on the $x$ and $y$ axes, respectively (Figure $2 \mathrm{c}$ ). ${ }^{29}$ The shaded yellow region in the middle of the diagram corresponds to properties that are suitable for inkjet printing. Regions toward the edges of the diagram are not suitable due to, for example, the likelihood of splashing on the substrate, formation of satellite drops, or clogging of the nozzle due to higher ink viscosity. Printability of inks can also be estimated via the reciprocal of the Ohnesorge number $\left(Z=1 / O_{\mathrm{h}}\right)$, with best printability in the range $10>Z>1 .{ }^{30}$ Too low $Z$ prevents printing due to too high pressure from the high viscosity, whereas too high $Z$ results in generation of satellite droplets. Based on fixed nozzle diameter of $21 \mu \mathrm{m}$ and droplet velocity of $7 \mathrm{~m} / \mathrm{s}$ (suitable values lie in the range from 6 to $10 \mathrm{~m} / \mathrm{s}$ ), we formulated printable inks by adjusting $R_{\mathrm{e}}$ and $O_{\mathrm{h}}$ (and $Z$ ) via the FAEG and surfactant concentrations. This enabled formulation of inks with properties in the center of the printing region of the phase diagram (blue square; $\eta=7 \mathrm{cP}, \gamma=28 \pm 2$ $\mathrm{mN} / \mathrm{m}, Z=3.0$ ). We also prepared less optimized inks with the single formamide solvent (red circle; $\eta=3 \mathrm{cP}, \gamma=28 \mathrm{mN}$ / $\mathrm{m}, Z=8.1$ ), for which printing was possible but resulted in satellite droplets (see Figure S2). We also note that droplet formation upon jetting is influenced by the applied waveform over the ink, which affects droplet ejection and shape. ${ }^{34}$ This is particularly relevant for our inks that contain NPs. Figure S3 shows customized waveform profiles for stable droplet ejection for the MNP inks.

Figure $2 \mathrm{~d}$ shows a schematic illustration of the MNP selfassembly process into microdomes after deposition on the substrate. After printing, solvent evaporation leads to shrinking 


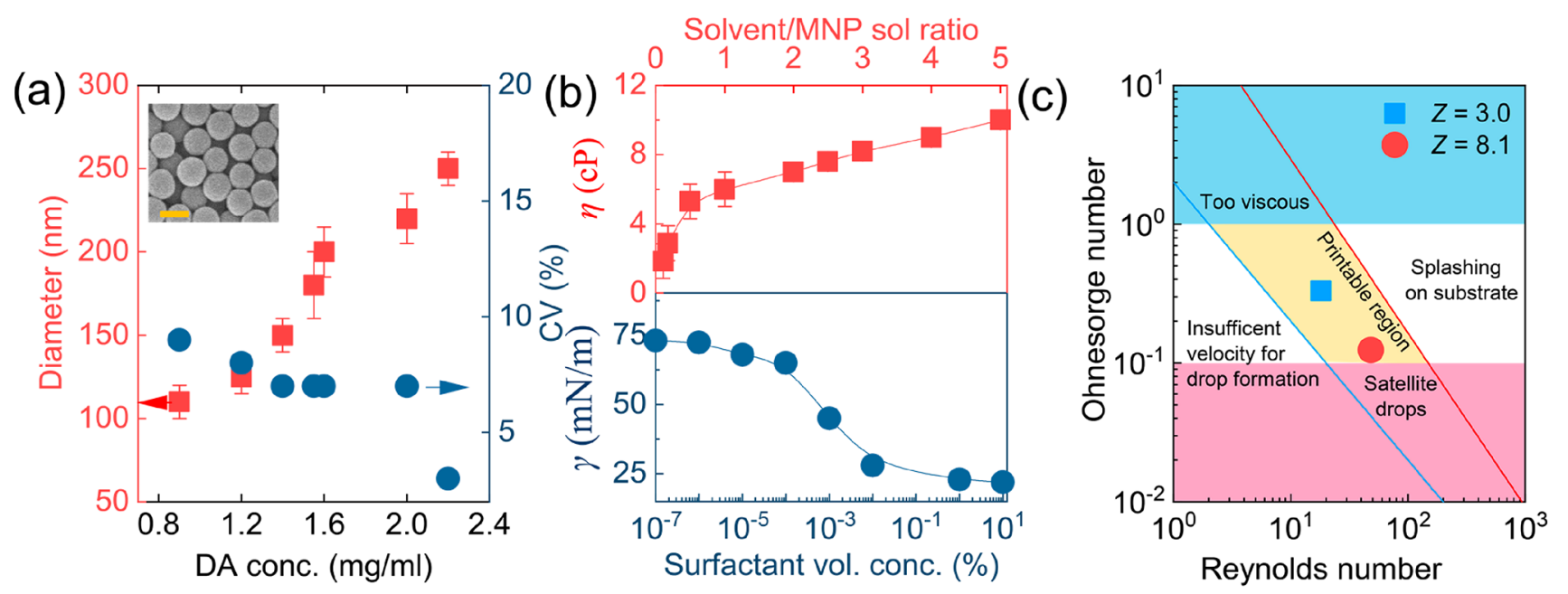

(d)

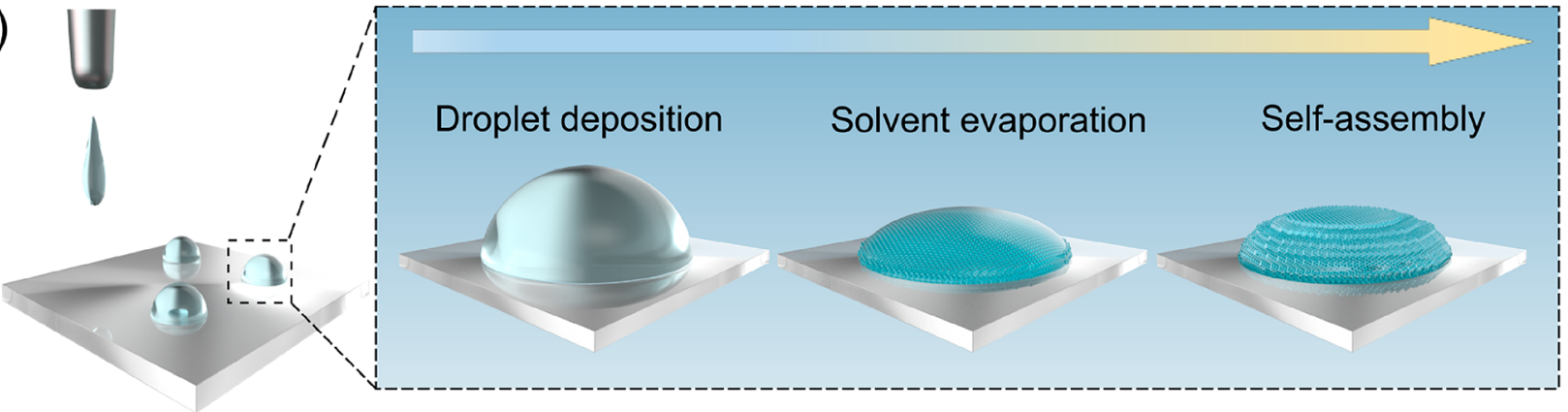

(e)
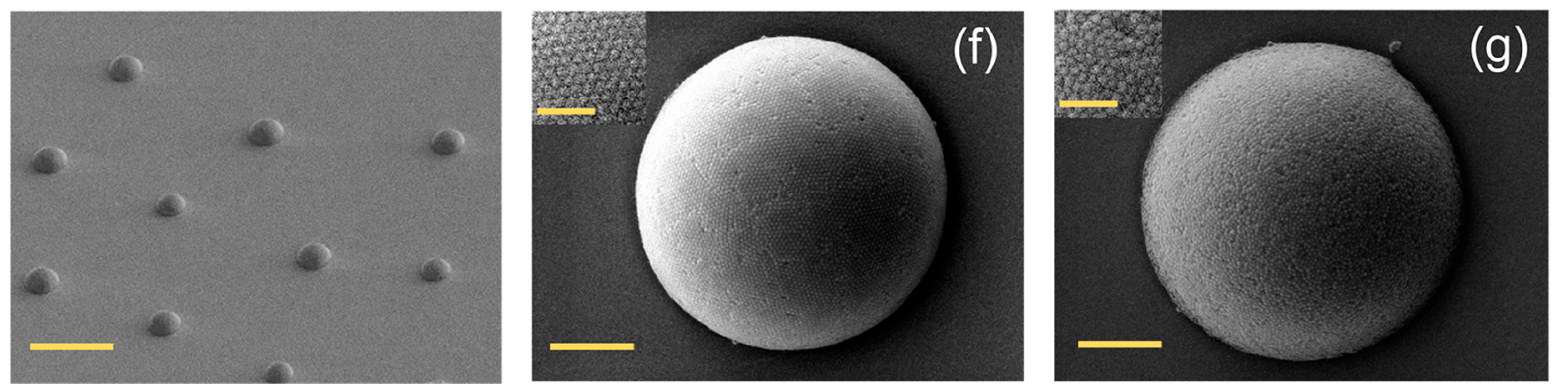

Figure 2. (a) Diameter and CV of MNPs as a function of monomer concentration. The inset shows an example of a SEM image of MNPs, as used to measure nanoparticle sizes (scale bar $=200 \mathrm{~nm}$ ). (b) Viscosity (top panel) and surface tension (bottom panel) of MNP inks as a function of solvent content and surfactant concentration, respectively. The top panel $x$ axis represents the ratio of solvent to MNP aqueous solution, using single solvent for ratios between 0 and 1 and binary solvents for larger ratios. (c) Phase diagram showing different operating regimes in inkjet printing. Inks with conditions optimized for both printing and ordered self-assembly are indicated with the blue square, whereas the red circle corresponds to printable ink, but less ordered MNP self-assembly. (d) Schematic visualization of inkjet printing and self-assembly of photonic MNP microdomes. (e) SEM image of several printed photonic MNP microdomes. The scale bar is $30 \mu \mathrm{m}$. (f) Representative high-magnification SEM micrographs of a photonic MNP microdome made using an optimized ink containing MNPs with diameter of $204 \mathrm{~nm}$. (g) Same as in (f), but using the non-optimized ink. The scale bars are $3 \mu \mathrm{m}$. The insets in $(\mathrm{f}, \mathrm{g})$ are magnified SEM images, with scale bars of $1 \mu \mathrm{m}$.

of the microdroplet while maintaining its dome shape. In this process, the dispersed MNPs come closer together, and when the MNP concentration reaches a critical value, they begin to crystallize, resulting in assembly of hemispherical microdomes. ${ }^{35}$ Figure 2e shows a SEM image of successful printing and assembly of MNP microdomes on a hydrophobic PDMS substrate (we used hydrophobic substrates to ensure high contact angles). These microdomes originate from printing of $10 \mathrm{pL}$ droplets of an optimized ink $(Z=3.0)$ containing 5 wt \% $204 \mathrm{~nm}$ in diameter MNPs, resulting in around $15 \mu \mathrm{m}$ hemispherical colloidal assemblies with high contact angle $\left(\sim 60^{\circ}\right)$. Closer inspection of a single microdome shows highly uniform, well-packed, and long-range-ordered MNPs, with crystalline patches resembling those of a soccer ball. Each large patch/domain shows hexagonally packed MNPs (Figure 2f). ${ }^{36,37}$ Grain boundaries and point defects on the surface of the microdomes can be attributed to stress and strain developed during the crystallization process, as discussed previously by Ling and co-workers for spherical assemblies of polystyrene microparticles. ${ }^{38}$ The average domain size was typically between 3 and $6 \mu \mathrm{m}$. To reveal the internal structure of the MNP microdomes, we cut them open with a focused ion beam (FIB) and imaged their interior by SEM (see Figure S4). All examined MNP microdomes consisted of MNP layers from top to bottom, without any hollow regions. Such a highly ordered multilayer MNP structure makes the microdomes promising for SC. 
(i)

(ii)

(iii)
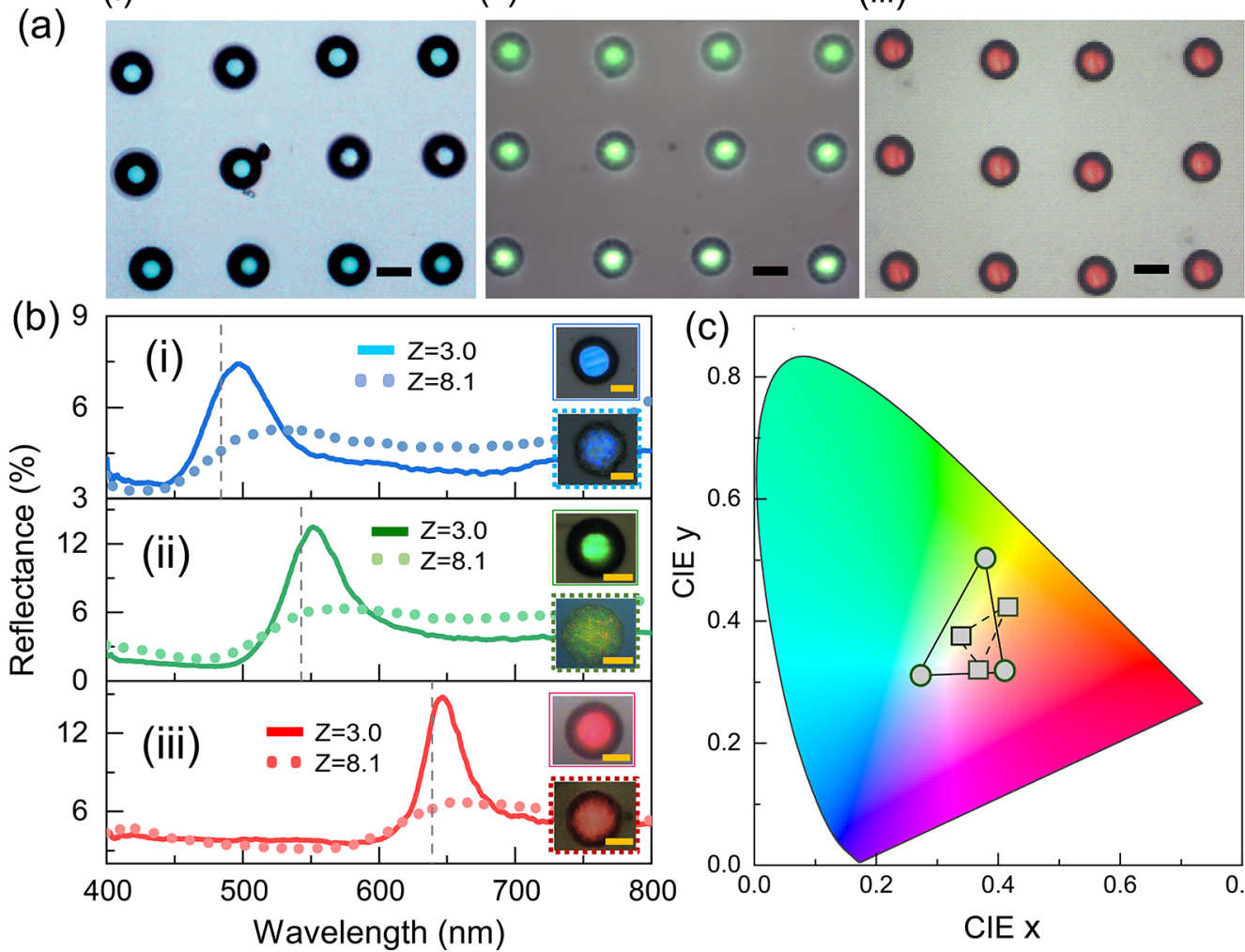

(b)

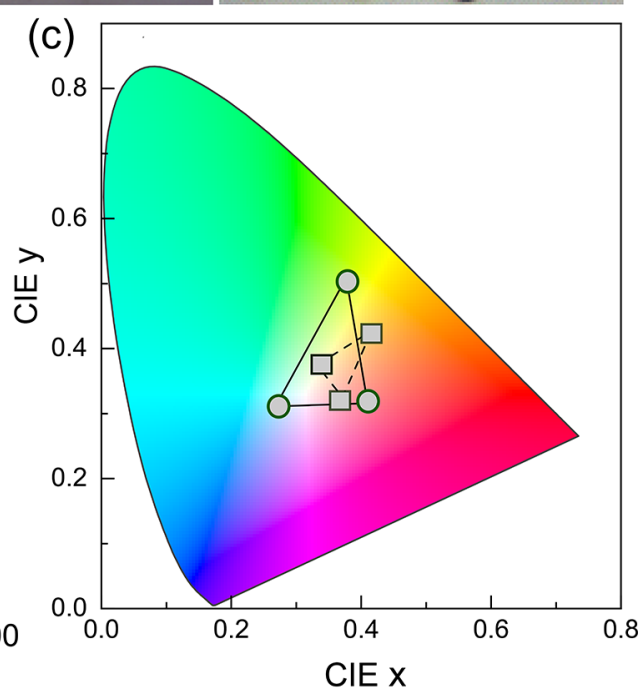

Figure 3. Optical properties of inkjet-printed photonic MNP microdomes. (a) Optical microscopy images of microdomes producing blue (i), green (ii), and red (iii) reflected SC, based on particles with diameter of 182, 204, and $240 \mathrm{~nm}$, respectively. The scale bars are $20 \mu \mathrm{m}$. (b) Reflection spectra and micrographs of single photonic MNP microdomes, made from optimized inks $(Z=3.0$, solid lines, top insets $)$ and non-optimized inks ( $Z=8.1$, dotted lines, bottom insets), with SC tunable across the visible spectrum. Particle diameters are the same as in (a). The scale bars are 10 $\mu \mathrm{m}$. (c) CIE chromaticity chart for reflected SC from single photonic MNP microdomes. The circles connected with solid lines correspond to the optimized photonic MNP microdomes, and the squares connected with dotted lines represent non-optimized photonic MNP microdomes.

It should be noted that ink printability is not sufficient to obtain microdomes with high MNP order. We demonstrate this by printing microdomes with inks with lower viscosity and higher Ohnesorge number $(Z=8.1)$, made using a single formamide solvent instead of the binary FAEG solvent. This non-optimized ink produced microdomes with a size similar to that for the optimized ink, but with non-closed-packed MNPs resembling colloidal photonic glass (Figure $2 \mathrm{~g}$ ). ${ }^{39}$ We attribute the lower order to the lower viscosity $(\sim 3 \mathrm{cP})$ and higher evaporation rate of this ink compared with the optimized ink, highlighting the importance of finding the suitable relation between MNP movements and microdroplet evaporation during the crystallization process.

Optical microscopy and microreflectance spectroscopy enabled us to reveal how MNP size and packing order influence the optical properties of the microdomes. Figure 3a shows optical microscopy images of microdome arrays printed on PDMS using optimized inks $(Z=3.0)$ containing MNPs of different sizes. The microdomes are uniform in size and shape and could be precisely positioned over large areas. The slight nonperfect alignment of the microdomes within the array could be related to the printing itself (e.g., varying jetting angle) or to contraction and movement of the droplets and particles during the self-assembly process. The microdomes all show strong reflective SC, with highly vivid colors and excellent uniformity between pixels. The different colors originate from MNPs of different diameters, enabling reproducible printing of red (i, $240 \mathrm{~nm}$ ), green (ii, $204 \mathrm{~nm}$ ), and blue (iii, $182 \mathrm{~nm}$ ) photonic MNP microdomes. Because every printed droplet results in an individual reflective photonic micropixel, the results are promising for printing images or for pixelated reflective displays.

The solid lines in Figure $3 \mathrm{~b}$ show reflection spectra of individual photonic microdomes made from optimized inks with MNP sizes of $182 \mathrm{~nm}$ (i, blue), $204 \mathrm{~nm}$ (ii, green), and $240 \mathrm{~nm}$ (iii, red). The top insets in each panel are optical images of the same pixels. They all show sharp reflection peaks (full width at half-maximum $\approx 50 \mathrm{~nm}$ ), resulting from Bragg diffraction by the highly ordered MNPs. The peak positions $\left(\lambda_{\max }\right)$ can be estimated from the Bragg-Snell law: ${ }^{40}$

$$
m \lambda_{\max }=2 d_{h k l} \sqrt{n_{\mathrm{eff}}^{2}-\sin _{\theta}^{2}}
$$

where $m$ is the diffraction order, $\lambda_{\max }$ is the diffracted wavelength, $n_{\text {eff }}$ is the effective refractive index, and $\theta$ is the incidence angle with respect to the normal. $d_{h k l}$ is the interplanar NP separation (see SI §1). The effective refractive index was estimated to be 1.63 (see SI §2). The dashed vertical lines in (Figure $3 \mathrm{~b}$ ) show the resulting calculated wavelengths, which are close to but slightly blue-shifted from the experimentally obtained peak positions.

Figure $3 \mathrm{~b}$ also presents the reflection spectra (dotted lines) and optical microscopy images (bottom insets in each panel) for single microdomes printed using the nonoptimized inks $(Z$ $=8.1$ ) but with same MNP diameters. Whereas these microdomes provide some SC, the colors are less pronounced and the reflection peaks are significantly broader and slightly 
(a)

a) Specular reflection

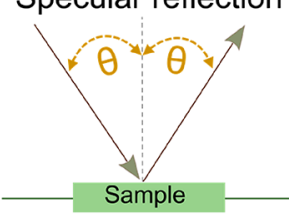

(b)
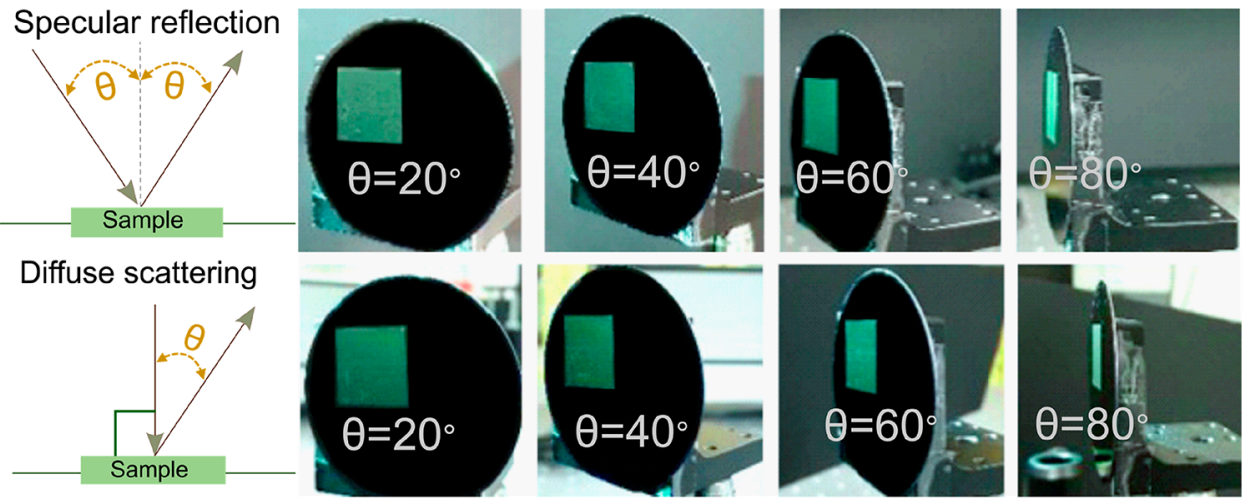

(c)
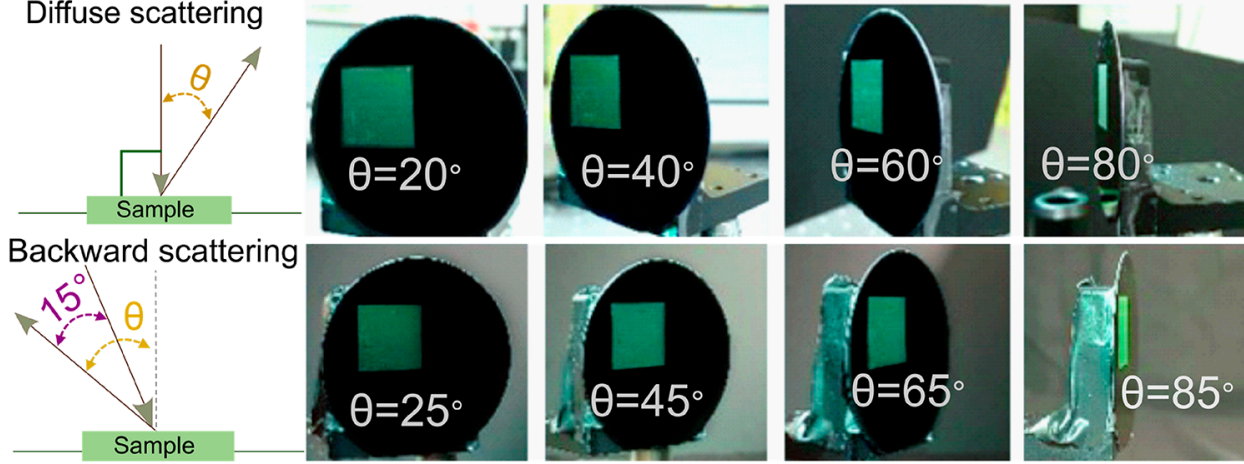

(d)
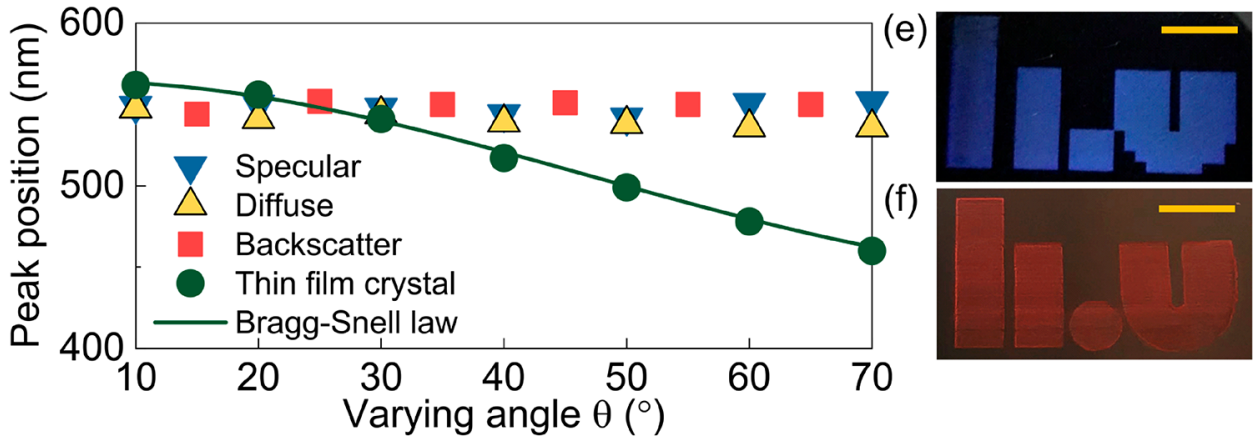

Figure 4. Noniridescence of inkjet printed photonic MNP microdome array made from $204 \mathrm{~nm}$ in diameter MNPs, printed on a Si substrate coated with a thin black PDMS layer (see SI). (a) Specular reflection: schematic of the measurement setup is shown to the left, followed by photographs at different incidence/detection angles. (b) Diffuse scattering: schematic of the measurement is shown to the left, followed by photographs at different detection angles. (c) Backward scattering: schematic of the measurement is shown to the left (only sample rotates), followed by photographs at different backscattering angles. (d) Reflection peak positions for the photonic microdome array at different angles obtained for specular (blue triangles), diffusive scattering (yellow triangles), and backward scattering (red squares). The green circles correspond to the flat photonic crystal control sample, and the green solid line corresponds to the predicted angle dependence from Bragg-Snell law. (e,f) Digital photographs of large-area blue and red prints inspired by our university logo (published with permission), printed on Si with black PDMS (e) and on black paper (f) and captured normal to the surface under ambient light conditions. The scale bars in (e,f) correspond to $1 \mathrm{~cm}$.

red-shifted compared to those with the photonic MNP domes printed using optimized inks. The SC for these microdomes also shows significant spatial variation across the single pixels. We attribute these effects to the nonuniform and amorphous nature of the MNP packing for these microdomes, which leads to less efficient Bragg diffraction and a red shift due to larger average interparticle separation.

To provide a more intuitive appreciation of the color hues of the photonic microdomes, we converted the measured reflectance spectra to data points in the Commission Internationale de L'Eclairage (CIE) chromaticity chart (Figure $3 c$ ). As expected, the optimized photonic microdomes (round markers, connected via the solid black lines) display colors with relatively high chromaticity that are tunable by MNP size. By contrast, the limited MNP order of the non-optimized microdomes seriously affected chromaticity and resulted in muted and pastel-like colors (square markers, connected by the dashed black lines).

Re-examining the optical microscope images of the optimized photonic MNP microdomes reveals uniform color in the center surrounded by a dark rim. This phenomenon is related to the microdome shape and that the angle of the photonic crystal lattice varies gradually across the microdomes.
In the center, the crystal lattice is parallel to the substrate, such that light at normal incidence will predominantly result in SC reflected back normal to the substrate. However, toward the rim of the domes, light incident normal to the substrate will experience a large incidence angle with respect to the photonic crystal lattice (up to around $60^{\circ}$ at the edges). Therefore, the dominant part of the diffracted light will not reflect back from where it came. To verify this explanation, we imaged the same microdomes using microscope objectives of different numerical apertures (NA). Lenses with higher NA collect light from larger angles and should therefore collect back-reflected SC from a wider range of angles than lenses with low NA. ${ }^{41}$ Indeed, we found that the size of the colored center area of the microdome increased with increasing collection angle (measured from 9 to $53^{\circ}$; see Figure S5). The fact that the increase was also gradual is consistent with gradual tilting of the crystal lattice across the domes, which is promising for achieving noniridescent SC.

Next, we demonstrate that the photonic MNP microdomes provide highly noniridescent SC and that they can be used to form large area surfaces with angle-independent reflected colors. We inkjet printed a $1 \times 1 \mathrm{~cm}^{2}$ square array of green photonic MNP microdomes (optimized ink with $204 \mathrm{~nm}$ 
MNPs, dome spacing of $40 \mu \mathrm{m}$ ) on a black substrate (Si wafer coated with thin layer of PDMS mixed with carbon nanotubes; see SI for details). In brief, the photonic microdome arrays provided vivid and highly noniridescent green SC for both specular reflection as well as for diffuse scattering and back scattering (see Figure 4). Figure 4a shows photographs taken for specular reflection at different angles, and the color remains essentially the same up to the largest incidence angle of $80^{\circ}$. Indeed, optical spectroscopy shows hardly any variation in the reflection peak position for different angles (blue triangles in Figure 4d, full spectra in Figure S6). By contrast, flat photonic crystal control samples made from the same MNPs (see SI for details) show significant angle dependence, following the Bragg-Snell law as expected (green circles and green full line in Figure 4d, respectively). As a result, flat photonic crystals completely change their appearance when viewed at different angles (see photographs in Figure S6c).

Many practical applications, such as reflective displays, rely on nonspecular scattering for a good user experience. We therefore investigated the diffuse scattering properties for our photonic MNP microdome array by varying the detection angle while maintaining the incident light normal to the substrate. From the corresponding photographs (Figure $4 \mathrm{~b}$ ) and spectral response (yellow triangles in Figure $4 \mathrm{~d}$, full spectra in Figure S6), we conclude that the diffusive scattering from the photonic domes is highly noniridescent. We observe clear green scattering all the way up to an $80^{\circ}$ detection angle. Similarly, the microdomes provide green back-scattered light (Figure $4 \mathrm{c}$, by rotating the sample and keeping a fixed $15^{\circ}$ angle between the light source and the detector), with minimal change in color for different angles (also see red squares in Figure $4 \mathrm{~d}$ ). The flat photonic crystal film provided only marginal diffusive and back scattering, highlighting the role of the microdome-shaped photonic crystals to obtain both noniridescence and nonspecular reflection. The results are consistent with results obtained for spherical NP assemblies (supraballs) $^{22}$ and microdomes made of polystyrene NPs. ${ }^{15}$ Although originating from single photonic MNP microdomes, the noniridescence of our surfaces translates to the macroscale properties of the arrays. To further highlight the possibility for reproducible printing of photonic MNP microdomes in welldefined patterns over large areas, we printed a $3 \times 1.8 \mathrm{~cm}$ image of our university logo using blue microdomes on black PDMS on Si (Figure 4e). We could also print large patterns with with vibrant SC on a black paper sheet (treated with octadecyltrichlorosilane to make it hydrophobic), although further work needs to investigate the exact shape and order of the MNP assemblies formed on the paper (Figure 4f). It was also possible to print images containing all three primary colors (see Figure S7), which could be further improved using printers with multiple cartridges to avoid need to change cartridges between colors.

In conclusion, we have demonstrated inkjet printing of MNPs and showed that they can assemble into microdomes that provide highly noniridescent SC with high chromaticity. This strategy supersedes conventional time-consuming selfassembly processes. MNP inks containing different NP sizes could be prepared and printed in accurate patterns on different substrates. Viscosity and surface tension of the inks played vital roles to both facilitate the printing and to obtain microdomes with high MNP order. Spectroscopic investigation of single photonic MNP microdomes showed that they behave as individual photonic crystals with spatially varying crystal orientation. Their structural color arise from Bragg diffraction, with colors tunable via MNP size. Our work highlights the advantages of fabricating biomimicking photonic pixels by inkjet printing, which can reduce processing times and material requirement compared with conventional self-assembly methods and provide the ability to create arbitrary patterns of noniridescent structural colors on a variety of substrates.

\section{ASSOCIATED CONTENT}

\section{Supporting Information}

The Supporting Information is available free of charge at https://pubs.acs.org/doi/10.1021/acs.nanolett.0c02604.

Materials and methods, including synthesis and characterization of MNPs, MNP ink formulation, preparation of thin film photonic crystals, and structural and optical characterization; supplementary figures show SEM image of a satellite droplet, jetting waveform, FIB cut of a photonic crystal microdome, optical images of single microdomes imaged at different collection angles, optical images of color prints containing microdomes of different colors, optical spectroscopy of microdomes, and thin film photonic crystals (PDF)

\section{AUTHOR INFORMATION}

\section{Corresponding Author}

Magnus P. Jonsson - Laboratory of Organic Electronics,

Linköping University, SE-601 74 Norrköping, Sweden;

(1) orcid.org/0000-0002-3002-3639;

Email: magnus.jonsson@liu.se

\section{Authors}

Ravi Shanker - Laboratory of Organic Electronics, Linköping University, SE-601 74 Norrköping, Sweden

Samim Sardar - Laboratory of Organic Electronics, Linköping University, SE-601 74 Norrköping, Sweden; Center for Nano Science and Technology@PoliMi, Istituto Italiano di Tecnologia (IIT), 20133 Milano, Italy

Shangzhi Chen - Laboratory of Organic Electronics, Linköping University, SE-601 74 Norrköping, Sweden; 이이.org/ 0000-0002-7410-2531

Sampath Gamage - Laboratory of Organic Electronics, Linköping University, SE-601 74 Norrköping, Sweden

Stefano Rossi - Laboratory of Organic Electronics, Linköping University, SE-601 74 Norrköping, Sweden

Complete contact information is available at: https://pubs.acs.org/10.1021/acs.nanolett.0c02604

\section{Author Contributions}

R.S. and M.J. conceived the original idea. R.S. carried out the material synthesis, characterization, inkjet printing, optical characterization, and interpreted the data. S.S. assisted in setting up the microspectroscopy setup and initial inkjet printing. S.C. performed FIB-SEM measurement. S.G. contributed to angle-dependent spectroscopic measurements, and S.R. contributed to calculations and theoretical understanding. All work was coordinated by M.J. R.S. and M.J. wrote the manuscript through contributions of all authors. All authors has approved the final version of the manuscript.

\section{Notes}

The authors declare no competing financial interest. 


\section{ACKNOWLEDGMENTS}

We acknowledge financial support from the Wenner-Gren Foundation; the Swedish Research Council; the Knut and Alice Wallenberg foundation, the Swedish Foundation for Strategic research; the Knut and Alice Wallenberg foundation, Linköping University, and industry through the Wallenberg Wood Science Center the Wallenberg Wood Science Centre; and the Swedish Government Strategic Research Area in Materials Science on Functional Materials at Linköping University (Faculty Grant SFO-Mat-LiU No. 2009 00971). The authors thank Evan Kang for preliminary SEM data, and Subimal Majee for help with Ceradrop inkjet printing at Printed Electronics Arena (PEA), Norrköping.

\section{REFERENCES}

(1) Vukusic, P.; Sambles, J. R. Photonic structures in biology. Nature 2003, 424 (6950), 852-855.

(2) Parker, A. R.; Townley, H. E. Biomimetics of photonic nanostructures. Nat. Nanotechnol. 2007, 2 (6), 347.

(3) Xiong, R.; Luan, J.; Kang, S.; Ye, C.; Singamaneni, S.; Tsukruk, V. V. Biopolymeric photonic structures: design, fabrication, and emerging applications. Chem. Soc. Rev. 2020, 49 (3), 983-1031.

(4) Wu, P.; Wang, J.; Jiang, L. Bio-inspired photonic crystal patterns. Mater. Horiz. 2020, 7, 338 .

(5) Nassau, K. The fifteen causes of color: the physics and chemistry of color. Color Res. Appl. 1987, 12 (1), 4-26.

(6) Kim, S.-H.; Lee, S. Y.; Yang, S.-M.; Yi, G.-R. Self-assembled colloidal structures for photonics. NPG Asia Mater. 2011, 3 (1), 2533.

(7) Xia, Y.; Gates, B.; Li, Z. Y. Self-assembly approaches to threedimensional photonic crystals. Adv. Mater. 2001, 13 (6), 409-413.

(8) von Freymann, G.; Kitaev, V.; Lotsch, B. V.; Ozin, G. A. Bottomup assembly of photonic crystals. Chem. Soc. Rev. 2013, 42 (7), 25282554.

(9) Zhang, J.; Sun, Z.; Yang, B. Self-assembly of photonic crystals from polymer colloids. Curr. Opin. Colloid Interface Sci. 2009, 14 (2), 103-114.

(10) Tan, A. T.; Nagelberg, S.; Chang-Davidson, E.; Tan, J.; Yang, J. K.; Kolle, M.; Hart, A. J. In-Plane Direct-Write Assembly of Iridescent Colloidal Crystals. Small 2020, 16 (4), 1905519.

(11) Kumar, K.; Duan, H.; Hegde, R. S.; Koh, S. C.; Wei, J. N.; Yang, J. K. Printing colour at the optical diffraction limit. Nat. Nanotechnol. 2012, 7 (9), 557-561.

(12) Ko, Y. G.; Shin, D. H. Effects of liquid bridge between colloidal spheres and evaporation temperature on fabrication of colloidal multilayers. J. Phys. Chem. B 2007, 111 (7), 1545-1551.

(13) Vogel, N.; Retsch, M.; Fustin, C.-A.; del Campo, A.; Jonas, U. Advances in colloidal assembly: the design of structure and hierarchy in two and three dimensions. Chem. Rev. 2015, 115 (13), 6265-6311.

(14) Shi, L.; Zhang, Y.; Dong, B.; Zhan, T.; Liu, X.; Zi, J. Amorphous Photonic Crystals with Only Short-Range Order. Adv. Mater. 2013, 25 (37), 5314-5320.

(15) Kuang, M.; Wang, J.; Bao, B.; Li, F.; Wang, L.; Jiang, L.; Song, Y. Inkjet printing patterned photonic crystal domes for wide viewingangle displays by controlling the sliding three phase contact line. Adv. Opt. Mater. 2014, 2 (1), 34-38.

(16) Yang, X.; Ge, D.; Wu, G.; Liao, Z.; Yang, S. Production of Structural Colors with High Contrast and Wide Viewing Angles from Assemblies of Polypyrrole Black Coated Polystyrene Nanoparticles. ACS Appl. Mater. Interfaces 2016, 8 (25), 16289-16295.

(17) Baumberg, J.; Pursiainen, O.; Spahn, P. Resonant optical scattering in nanoparticle-doped polymer photonic crystals. Phys. Rev. B: Condens. Matter Mater. Phys. 2009, 80 (20), 201103.

(18) Aguirre, C. I.; Reguera, E.; Stein, A. Colloidal photonic crystal pigments with low angle dependence. ACS Appl. Mater. Interfaces 2010, 2 (11), 3257-3262.
(19) Takeoka, Y.; Yoshioka, S.; Takano, A.; Arai, S.; Nueangnoraj, K.; Nishihara, H.; Teshima, M.; Ohtsuka, Y.; Seki, T. Production of colored pigments with amorphous arrays of black and white colloidal particles. Angew. Chem., Int. Ed. 2013, 52 (28), 7261-7265.

(20) Kohri, M.; Nannichi, Y.; Taniguchi, T.; Kishikawa, K. Biomimetic non-iridescent structural color materials from polydopamine black particles that mimic melanin granules. J. Mater. Chem. C 2015, 3 (4), 720-724.

(21) Xiao, M.; Li, Y.; Allen, M. C.; Deheyn, D. D.; Yue, X.; Zhao, J.; Gianneschi, N. C.; Shawkey, M. D.; Dhinojwala, A. Bio-inspired structural colors produced via self-assembly of synthetic melanin nanoparticles. ACS Nano 2015, 9 (5), 5454-5460.

(22) Xiao, M.; Hu, Z.; Wang, Z.; Li, Y.; Tormo, A. D.; Le Thomas, N.; Wang, B.; Gianneschi, N. C.; Shawkey, M. D.; Dhinojwala, A. Bioinspired bright noniridescent photonic melanin supraballs. Science advances 2017, 3 (9), No. e1701151.

(23) Eliason, C. M.; Shawkey, M. D. A photonic heterostructure produces diverse iridescent colours in duck wing patches. J. R. Soc., Interface 2012, 9 (74), 2279-2289.

(24) Eliason, C. M.; Shawkey, M. D.; Clarke, J. A. Evolutionary shifts in the melanin-based color system of birds. Evolution 2016, 70 (2), $445-455$.

(25) Cui, L.; Li, Y.; Wang, J.; Tian, E.; Zhang, X.; Zhang, Y.; Song, Y.; Jiang, L. Fabrication of large-area patterned photonic crystals by ink-jet printing. J. Mater. Chem. 2009, 19 (31), 5499-5502.

(26) Fenzl, C.; Hirsch, T.; Wolfbeis, O. S. Photonic crystals for chemical sensing and biosensing. Angew. Chem., Int. Ed. 2014, 53 (13), 3318-3335.

(27) Sardar, S.; Wojcik, P.; Kang, E. S.; Shanker, R.; Jonsson, M. P. Structural coloration by inkjet-printing of optical microcavities and metasurfaces. J. Mater. Chem. C 2019, 7 (28), 8698-8704.

(28) Doncom, K. E.; Blackman, L. D.; Wright, D. B.; Gibson, M. I.; O’Reilly, R. K. Dispersity effects in polymer self-assemblies: a matter of hierarchical control. Chem. Soc. Rev. 2017, 46 (14), 4119-4134.

(29) Derby, B. Inkjet printing of functional and structural materials: fluid property requirements, feature stability, and resolution. Annu. Rev. Mater. Res. 2010, 40, 395-414.

(30) Jang, D.; Kim, D.; Moon, J. Influence of fluid physical properties on ink-jet printability. Langmuir 2009, 25 (5), 2629-2635.

(31) Lau, C. Y.; Duan, H.; Wang, F.; He, C. B.; Low, H. Y.; Yang, J. $\mathrm{K}$. Enhanced ordering in gold nanoparticles self-assembly through excess free ligands. Langmuir 2011, 27 (7), 3355-3360.

(32) Asbahi, M.; Mehraeen, S.; Wang, F.; Yakovlev, N.; Chong, K. S.; Cao, J.; Tan, M. C.; Yang, J. K. Large area directed self-assembly of sub-10 nm particles with single particle positioning resolution. Nano Lett. 2015, 15 (9), 6066-6070.

(33) Zhang, Y.; Qian, Y.; Liu, Z.; Li, Z.; Zang, D. Surface wrinkling and cracking dynamics in the drying of colloidal droplets. Eur. Phys. J. E: Soft Matter Biol. Phys. 2014, 37 (9), 84.

(34) Dong, H.; Carr, W. W.; Morris, J. F. An experimental study of drop-on-demand drop formation. Phys. Fluids 2006, 18 (7), No. 072102.

(35) Das, S.; Dey, A.; Reddy, G.; Sarma, D. Suppression of the coffee-ring effect and evaporation-driven disorder to order transition in colloidal droplets. J. Phys. Chem. Lett. 2017, 8 (19), 4704-4709.

(36) Velev, O. D.; Lenhoff, A. M.; Kaler, E. W. A Class of Microstructured Particles Through Colloidal Crystallization. Science 2000, 287 (5461), 2240-2243.

(37) Manoharan, V. N.; Elsesser, M. T.; Pine, D. J. Dense Packing and Symmetry in Small Clusters of Microspheres. Science 2003, 301 (5632), 483-487.

(38) Ling, X. S. Scars on a colloidal crystal ball. Nat. Mater. 2005, 4 (5), 360-361.

(39) García, P. D.; Sapienza, R.; Blanco, Á; López, C. Photonic glass: a novel random material for light. Adv. Mater. 2007, 19 (18), 25972602.

(40) Gonzalez-Urbina, L.; Baert, K.; Kolaric, B.; Perez-Moreno, J.; Clays, K. Linear and nonlinear optical properties of colloidal photonic crystals. Chem. Rev. 2012, 112 (4), 2268-2285. 
(41) Vogel, N.; Utech, S.; England, G. T.; Shirman, T.; Phillips, K.

R.; Koay, N.; Burgess, I. B.; Kolle, M.; Weitz, D. A.; Aizenberg, J.

Color from hierarchy: Diverse optical properties of micron-sized spherical colloidal assemblies. Proc. Natl. Acad. Sci. U. S. A. 2015, 112 (35), 10845-10850. 